\title{
475.
}

\section{ON A PROPERTY OF THE STEREOGRAPHIC PROJECTION.}

[From the Monthly Notices of the Royal Astronomical Society, vol. xxx. (1869-1870), pp. 205-207.]

I AM not aware whether it has been noticed that the very same circles which in the direct stereographic projection of a hemisphere (viz., that wherein the projection is on the plane of a meridian) represent the meridians and parallels respectively,represent also in the oblique projection of the hemisphere meridians and parallels respectively. In fact, in the direct projection where the poles $N, S$, are in the horizon-meridian, or bounding circle of the projection, if we take a chord $A B$ at right angles to $N S$, and on $A B$ as diameter describe a circle, the original (meridian and parallel) circles will, as the appearance of the figure at once suggests, represent meridians and parallels in the oblique projection in which the horizon or bounding circle of the projection is the circle diameter $A B$, and where consequently the North Pole $N$ is brought into view, the South Pole $S$ being beyond the limits of the projection. That this really is so, is clear from the consideration that in any stereographic projection whatever, the meridians will be circles passing through two fixed points $N, S$, and the parallels be circles cutting the meridians at right angles. (Or, what is the same thing, the parallels also pass each of them through two fixed imaginary points, the antipoints of $N, S$, but this in passing.) And moreover since in the oblique, as well as in the direct, projection, the longitude of any meridian, as reckoned from the central meridian $N S$, is the angle at $N$ between the two meridians, the longitude for a given meridian is the same in the two projections respectively. But the co-latitudes are not the same in the two projections respectively; viz., a circle which in the direct projection represents the parallel co-latitude $c$, will in the oblique projection represent the parallel of a different co-latitude $c^{\prime}$. The relation between the values of $c, c^{\prime}$, will of course depend upon the position of the bounding 
circle $A B$ of the oblique direction: to define this position, we may use either the are $N M$ which in the direct projection determines the co-latitude of the centre $M$ of the oblique projection (say $N M=\Delta$, that is, $N V=\Delta$ ), or by the arc $N M$ which in the oblique projection determines the distance of $N$ from the centre, or co-latitude of the

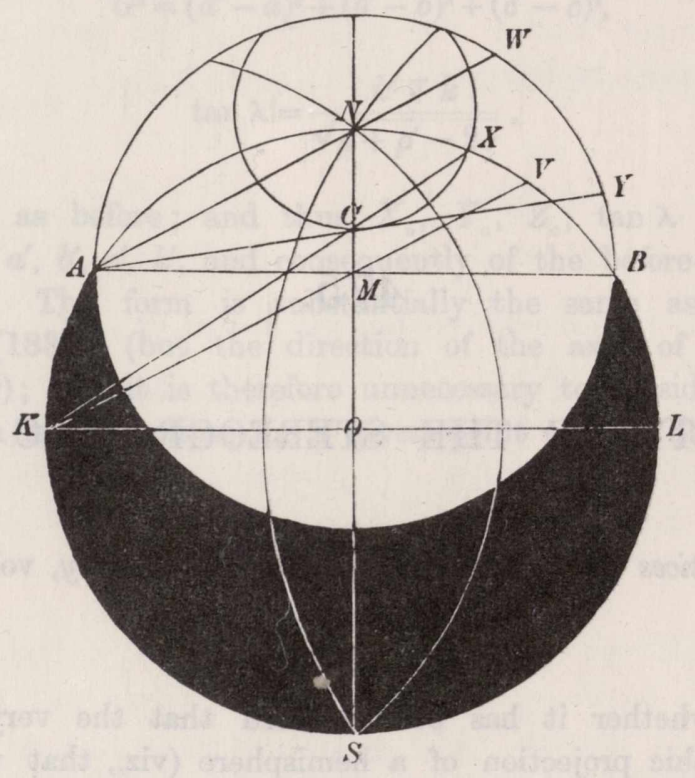

centre (say $N M=\Delta^{\prime}$, that is, $B W=\Delta^{\prime}$ ). The obliquity in the oblique projection is thus $90^{\circ}-\Delta^{\prime}$, viz., this is the inclination of the plane of projection to that of the horizonmeridian in the direct projection. We have also $c=N X, c^{\prime}=W Y$. The relation between the angles $\Delta, \Delta^{\prime}$, is easily found to be

$$
\tan \frac{1}{2} \Delta=\tan ^{2} \frac{1}{2} \Delta^{\prime}
$$

viz., taking the radius in the direct projection to $b e=1$, we have

$$
\begin{aligned}
& O M=\tan \frac{1}{2}\left(90^{\circ}-\Delta\right), \\
& M A=\sqrt{1-\tan ^{2} \frac{1}{2}\left(90^{\circ}-\Delta\right),} \\
& M N=1-\tan \frac{1}{2}\left(90^{\circ}-\Delta\right) ;
\end{aligned}
$$

wherefore

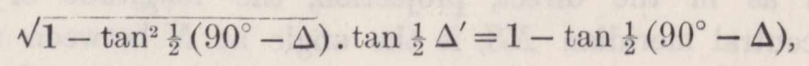

and thence

$$
\tan ^{2} \frac{1}{2} \Delta^{\prime}=\frac{1-\tan \frac{1}{2}\left(90^{\circ}-\Delta\right)}{1+\tan \frac{1}{2}\left(90^{\circ}-\Delta\right)}=\tan \frac{1}{2} \Delta
$$

the required relation. 
We have moreover

$$
\begin{aligned}
N C=1-\tan \frac{1}{2}\left(90^{\circ}-c\right) & =A M \quad\left\{\tan \frac{1}{2} \Delta^{\prime}-\tan \frac{1}{2}\left(\Delta^{\prime}-c^{\prime}\right)\right\} \\
& =\sin \Delta^{\prime}\left\{\tan \frac{1}{2} \Delta^{\prime}-\tan \frac{1}{2}\left(\Delta^{\prime}-c^{\prime}\right)\right\} \\
& =2 \sin ^{2} \frac{1}{2} \Delta^{\prime}-\sin \Delta^{\prime} \tan \frac{1}{2}\left(\Delta^{\prime}-c^{\prime}\right)
\end{aligned}
$$

that is

$$
\begin{aligned}
\tan \frac{1}{2}\left(90^{\circ}-c\right) & =\cos \Delta^{\prime}+\sin \Delta^{\prime} \tan \frac{1}{2}\left(\Delta^{\prime}-c^{\prime}\right), \\
& =\frac{\cos \frac{1}{2}\left(\Delta^{\prime}+c^{\prime}\right)}{\cos \frac{1}{2}\left(\Delta^{\prime}-c^{\prime}\right)}
\end{aligned}
$$

or, what is the same thing,

that is

$$
\frac{1-\tan \frac{1}{2} c}{1+\tan \frac{1}{2} c}=\frac{1+\delta \tan \frac{1}{2} c^{\prime} \tan \frac{1}{2} \Delta^{\prime}}{1+\tan \frac{1}{2} c^{\prime} \tan \frac{1}{2} \Delta^{\prime}}
$$

$$
\tan \frac{1}{2} c=\tan \frac{1}{2} \Delta^{\prime} \tan \frac{1}{2} c^{\prime}
$$

which is the required relation between $c$ and $c^{\prime}$. In the particular case $\Delta=\Delta^{\prime}=90^{\circ}$, the two projections coincide, and we have, as we should do, $c^{\prime}=c$. 